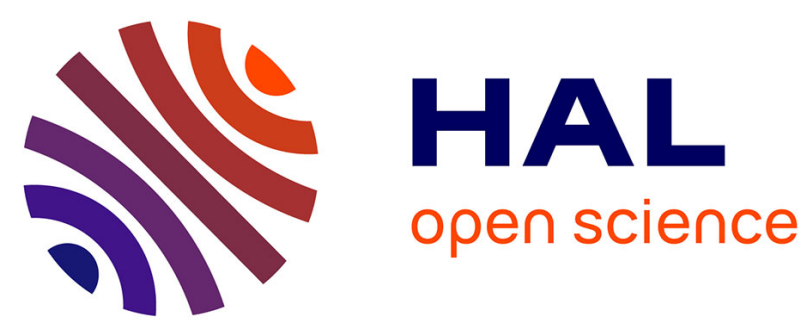

\title{
Respective contribution of ictal and inter-ictal electrical source imaging to epileptogenic zone localization
}

Estelle Rikir, Louis G Maillard, Chifaou Abdallah, Martine Gavaret, Fabrice Bartolomei, Jean-Pierre Vignal, Sophie Colnat-Coulbois, Laurent Koessler, Christoph M Michel

\section{To cite this version:}

Estelle Rikir, Louis G Maillard, Chifaou Abdallah, Martine Gavaret, Fabrice Bartolomei, et al.. Respective contribution of ictal and inter-ictal electrical source imaging to epileptogenic zone localization. Brain Topography: a Journal of Cerebral Function and Dynamics, 2020, 33 (3), pp.384-402. 10.1007/s10548-020-00768-3 . hal-02931212

\section{HAL Id: hal-02931212 \\ https://hal.science/hal-02931212}

Submitted on 23 Nov 2020

HAL is a multi-disciplinary open access archive for the deposit and dissemination of scientific research documents, whether they are published or not. The documents may come from teaching and research institutions in France or abroad, or from public or private research centers.
L'archive ouverte pluridisciplinaire HAL, est destinée au dépôt et à la diffusion de documents scientifiques de niveau recherche, publiés ou non, émanant des établissements d'enseignement et de recherche français ou étrangers, des laboratoires publics ou privés. 


\title{
Respective Contribution of Ictal and Inter-ictal Electrical Source Imaging to Epileptogenic Zone Localization
}

\author{
Estelle Rikir $^{1,2,3} \cdot$ Louis G. Maillard $^{1,4} \cdot$ Chifaou Abdallah $^{1} \cdot$ Martine Gavaret $^{5,6} \cdot$ Fabrice Bartolomei $^{5,6}$. \\ Jean-Pierre Vignal ${ }^{1,4}$. Sophie Colnat-Coulbois ${ }^{4,7} \cdot$ Laurent Koessler $^{1,4}$
}

Received: 18 October 2019 / Accepted: 7 April 2020

○) Springer Science+Business Media, LLC, part of Springer Nature 2020

\begin{abstract}
Interictal electrical source imaging (ESI) encompasses a risk of false localization due to complex relationships between irritative and epileptogenic networks. This study aimed to compare the localizing value of ESI derived from ictal and inter-ictal EEG discharges and to evaluate the localizing value of ESI according to three different subgroups: MRI lesion, presumed etiology and morphology of ictal EEG pattern. We prospectively analyzed 54 of 78 enrolled patients undergoing pre-surgical investigation for refractory epilepsy. Ictal and inter-ictal ESI results were interpreted blinded to- and subsequently compared with stereoelectroencephalography as a reference method. Anatomical concordance was assessed at a sub-lobar level. Sensitivity and specificity of ictal, inter-ictal and ictal plus inter-ictal ESI were calculated and compared according to the different subgroups. Inter-ictal and ictal ESI sensitivity (84\% and 75\% respectively) and specificity (38\% and $50 \%$ respectively) were not statistically different. Regarding the sensitivity, ictal ESI was never higher than inter-ictal ESI. Regarding the specificity, ictal ESI was higher than inter-ictal ESI in malformations of cortical development (MCD) (60\% vs. $43 \%$ ) and in MRI positive patients (49\% vs. 30\%). Within the ictal ESI analysis, we showed a higher specificity for ictal spikes (59\%) and rhythmic discharges $>13 \mathrm{~Hz}(50 \%)$ than rhythmic discharges $<13 \mathrm{~Hz}(37 \%)$ and (ii) for MCD (60\%) than in other etiologies (29\%). This prospective study demonstrates the relevance of a combined interpretation of distinct interictal and ictal analysis. Inter-ictal analysis gave the highest sensitivity. Ictal analysis gave the highest specificity especially in patients with MCD or a lesion on MRI.
\end{abstract}

Keywords Electrical source imaging $\cdot$ Ictal discharges $\cdot$ HR-EEG $\cdot$ SEEG $\cdot$ Epilepsy surgery $\cdot$ Malformation of cortical development

\section{Introduction}

Handling Editor: Christoph M. Michel.

Laurent Koessler

laurent.koessler@univ-lorraine.fr

1 Université de Lorraine, CHRU-Nancy, Service de Neurologie, Nancy, France

2 CHRU Sart-Tilman, Service de Neurologie, Liège, Belgium

3 Faculté de Médecine, Université de Liège, Liège, Belgium

4 Université de Lorraine, CNRS, CRAN, Nancy, France

5 AP-HM, Service de Neurophysiologie Clinique, Hôpital de La Timone, Marseille, France

6 INSERM UMR 1106, Faculté de Médecine, Institut des Neurosciences Des Systèmes, Marseille, France

7 Université de Lorraine, CHRU Nancy, Service de Neurochirurgie, Nancy, France
Pre-surgical evaluation of focal refractory epilepsy aims to localize and delineate the cortical region primarily involved in the generation of seizures i.e. the epileptogenic zone (EZ). Full resection of the EZ remains the main predictor of seizure freedom after surgery (Tonini et al. 2004). Precise estimation of the EZ still often requires intracranial EEG (iEEG) or intracerebral EEG (SEEG) recordings. SEEG targets are currently defined based on electro-clinical hypothesis derived from inter-ictal but mainly from ictal video-EEG recordings and structural abnormalities on magnetic resonance imaging (MRI) (Maillard et al. 2017).

During the last two decades, several studies have demonstrated the accuracy of non-invasive electro-magnetic source imaging techniques (Michel et al. 2004; Brodbeck et al. 2011) and have assessed their clinical usefulness compared 
to standard pre-surgical evaluation (Knowlton et al. 2009; Beniczky et al. 2013; Rikir et al. 2014; Abdallah et al. 2017). Most of them used inter-ictal discharges (IID) for source localization (Gavaret et al. 2004, 2006, 2009; Brodbeck et al. 2011) because these discharges are easier to record during a limited period of time, have a higher signal-to-noise ratio (SNR), and are less susceptible to be contaminated by muscle and movement artifacts.

However, SEEG recordings have shown that the irritative zone (IZ) defined as the cortical areas generating IID is often larger than the EZ (Talairach and Bancaud 1966; Alarcon et al. 1997; Kahane et al. 2006). A recent SEEG study showed only a $56 \%$ concordance between the cortical areas displaying the maximal inter-ictal activity and the EZ (Bartolomei et al. 2016). This study compared the anatomical concordance (at the level of the brain structures) between the intracerebral SEEG contacts where interictal and ictal discharges were recorded. Simultaneous scalp and SEEG studies also proved that IID recorded in scalp electrodes could match with inter-ictal activity generated in cortical areas related to but outside of the EZ (Alarcon et al. 1994; Koessler et al. 2015). In previous ESI studies, we also demonstrated that localizing sources from IID encompasses a significant risk of localizing outside the EZ (Rikir et al. 2014; Abdallah et al. 2017).

In pre-surgical workup, scalp ictal (i.e. seizures) recordings remain a crucial and almost mandatory step for planning the invasive recordings (such as intracerebral electrodes placement) as well as the surgery. Therefore, it seems theoretically more relevant to apply source imaging techniques on ictal discharges because the ictal source localizations are expected to be more predictive of the EZ than those performed on IID.

Because of methodological constraints, few studies have assessed the localizing value of electrical or magnetic source imaging (ESI, MSI) on ictal discharges. They were often retrospective with either a limited number of cases or electrodes $(n=31,32$ channels: Boon et al. 2002; $n=10,64$ channels: Koessler et al. 2010; $n=12,32$ to 64 channels: Medvedovsky et al. 2012; $\mathrm{n}=13,54$ channels: Pellegrino et al. 2016; $n=14,32-204$ channels: Nemtsas et al. 2017; $\mathrm{n}=5$; 32-204 channels: Staljanssens et al. 2017; $\mathrm{n}=12$, 256 channels: Kuo et al. 2018; $\mathrm{n}=28,25$ channels: Koren et al. 2018; $\mathrm{n}=87,25$ channels: Sharma et al. 2018). These studies showed that ictal source imaging was feasible and yielded overall accurate source localization by comparison to inter-ictal ESI or other functional modalities like positron emission tomography (PET), single photon emission computed tomography (SPECT), and MRI. However, an additional question still remains concerning the respective contribution of ictal and inter-ictal ESI in different patient subgroups. In epilepsy surgery investigation, new functional brain modalities and their respective added value need to be investigated across different patient subgroups in order to define which exploration would be best suited and how to interpret them.

The purposes of our study were to prospectively evaluate and compare the respective contribution (sensitivity and specificity) of electrical source imaging derived from ictal and inter-ictal EEG discharges to localize the EZ using SEEG as the reference method. It is important to note, that we used the anatomo-electro-clinical definition of the EZ which refers to the site of primary organization of ictal discharge (Bancaud et al. 1970), encompassing the transition from inter ictal to ictal discharge (Grinenko et al. 2018), the initial discharge and early propagation in spontaneous seizures (Kahane et al. 2006). This practical definition is different from the theoretical definition of the EZ that refers to the necessary minimal resection volume to reach seizure freedom (Lüders et al. 2006). We further evaluated and compared ictal ESI results in different subgroups classified according to the morphology of the ictal pattern, the presence or absence of a MRI lesion and the presumed etiology.

\section{Materials and Methods}

\section{Patient Selection}

Seventy-eight consecutive patients with drug-resistant focal epilepsy were prospectively enrolled in the multi-centric National Clinical Research Project PHRC 2009-17-05, Clinical trial NCT 01090934 (Nancy, Marseille, and Reims).

Inclusion criteria were (1) age $>15$ years and (2) indication of SEEG recordings. We excluded patients with a (1) contraindication to SEEG, (2) indication of direct cortectomy according to non-invasive pre-surgical evaluation, and (3) MRI suggesting a progressive lesion. This study was approved by the ethics committee CPP Est III and all patients gave their informed written consent.

\section{Non-invasive Evaluation}

Non-invasive evaluation included comprehensive medical history, neurological examination, long-term VIDEO-EEG recordings, high-resolution MRI, and neuropsychological evaluation in all cases, as well as optional inter-ictal PET and/or inter-ictal/ictal SPECT.

\section{MRI Acquisition}

Structural MRI was acquired with a 1.5 or 3T Signa General Electric Medical System (Healthcare, Milwaukee, WI, USA) according to a standardized epilepsy protocol (Duncan 2003). MRI scans were reviewed in a multidisciplinary case 
management conference by experienced epileptologists and neuro-radiologists.

\section{4-Channel Scalp VIDEO-EEG Recordings and ESI}

\section{EEG Recordings}

EEG was recorded from 64-scalp taped electrodes, placed according to an adapted 10/10 system including four electrodes in the inferior temporal chain (FT10/9, P10/9) (Oostenveld and Praamstra 2001; Koessler et al. 2009; Seeck et al. 2017). The localization of all 64 electrodes and of three fiducials (nasion, right and left tragi) was performed prior to acquisition either with a three-dimensional digitizer system (3Space Fastrak; Polhemus, Colchester, VT, U.S.A.) or with an automated detection and labeling system of MRI visible sensors (ALLES) (Koessler et al. 2008). Electrode-skin impedance was below $5 \mathrm{k} \Omega$. EEG was recorded with a $1024 \mathrm{~Hz}$ sampling rate and a $0.53-400 \mathrm{~Hz}$ band-pass filter (University Hospital Nancy: Micromed, Italy; University Hospital Marseille: Deltamed, USA). The Fpz electrode generally provided the reference, except for frontal lobe cases, where the $\mathrm{Oz}$ electrode was used. Hyperventilation trials combined with anti-epileptic drug (AED) tapering served to activate inter-ictal discharges and seizures (Jonas et al. 2011). AED withdrawal appears to have no significant effect on ictal EEG manifestations (So and Gotman 1990). EEG, combined with HD-video, was recorded for 4 days, $24 \mathrm{~h}$ per day, in order to study inter-ictal and ictal discharges.

\section{Seizure Selection and Analysis}

In case of several recorded seizures (range 0-30), one representative seizure was selected for ESI analysis according to the following criteria: (i) ability of drawing up electroclinical correlations (seizure with patient out of video camera field excluded), (ii) absence of important artifacts. When electro-clinical patterns were different, one seizure of each type was selected for source localization.

Each selected seizure was visually analyzed by trained epileptologists (ER, LGM, MG and FB) using bipolar and monopolar montages. Ictal EEG discharges were marked and classified according to four morphological patterns adapted from those proposed by Foldvary et al. (2001): (1) ictal spike (IS); (2) rhythmic discharge (RD) $>13 \mathrm{~Hz}$; (3) RD $<13 \mathrm{~Hz}$ and (4) flattening.

Their occurrence was chronologically noted (first, second, third ictal EEG pattern) as well as their occurrence compared to the first objective clinical ictal discharge (before or after clinical onset). Channels that recorded ictal EEG discharges with maximal amplitude (dominant channels) were determined with Advanced Signal Analysis (ASA) software (ANT Software, Enschede, Netherlands). SNR was defined by dividing the highest ictal discharge amplitude by the highest background activity amplitude.

Temporal windows of analysis for each ictal EEG pattern ran from the onset to the end of the activity i.e. from a few milliseconds for ictal spikes to one or several seconds for rhythmic discharges. ESI was performed on the earliest ictal EEG pattern in the temporal domain. Flattening patterns were not considered for analysis as they do not seem to be of localizing value (Alarcon et al. 1995). In these cases, source localization was performed on the next ictal EEG pattern.

\section{Inter-ictal Discharge Detection and Analysis}

Two hours were selected for inter-ictal ESI after careful visual analysis of the full recording according to the following criteria: (i) absence of artifacts, (ii) presence of calm wakefulness, (iii) presence of IIDs representative of all IID types. Inter-ictal spikes $(<70 \mathrm{~ms})$ and sharp waves $(<200 \mathrm{~ms})$ were visually identified and marked in an average reference montage by one of four experienced epileptologists according to the following established criteria: (1) paroxysmal occurrence, (2) abrupt change in polarity, (3) duration $<200 \mathrm{~ms}$, and (4) scalp topography consistent with a physiologic field. Temporal windows of analysis ( $\max 100 \mathrm{~ms}$ ) were defined around the IID and centered at the time of maximal negativity on the electrode trace with the highest amplitude. All 64-electrode traces were then super imposed to ascertain that the SNR, defined as the highest IID amplitude divided by the highest background activity amplitude, was $>2.5$. For each patient, we selected an average of 15 single IIDs. Then, we categorized them according to their respective topography and morphology (range 1-4 IID types, average of 2 IID types by patient), and finally we performed the source localization on the single IIDS (Fig. 1).

\section{Volume Conduction Parameters}

Using individual MRI, we chose to construct realistic head models (Boundary Element Model) for each patient due to evidence for lower ESI accuracy with spherical head models derived from a template MRI (Brodbeck et al. 2011; Guggisberg et al. 2011). This is described in detail in Rikir et al. (2014). Scalp, skull and brain tissue conductivities were respectively set at $0.33,0.04$ and $0.33 \mathrm{~S} / \mathrm{m}$ (Koessler et al. 2017).

\section{Inverse Problem Methods}

Intra-cerebral sources of each selected ictal and inter-ictal discharge were modeled by both equivalent current dipole (ECD) (Scherg 1990), a Multiple SIgnal Classification 


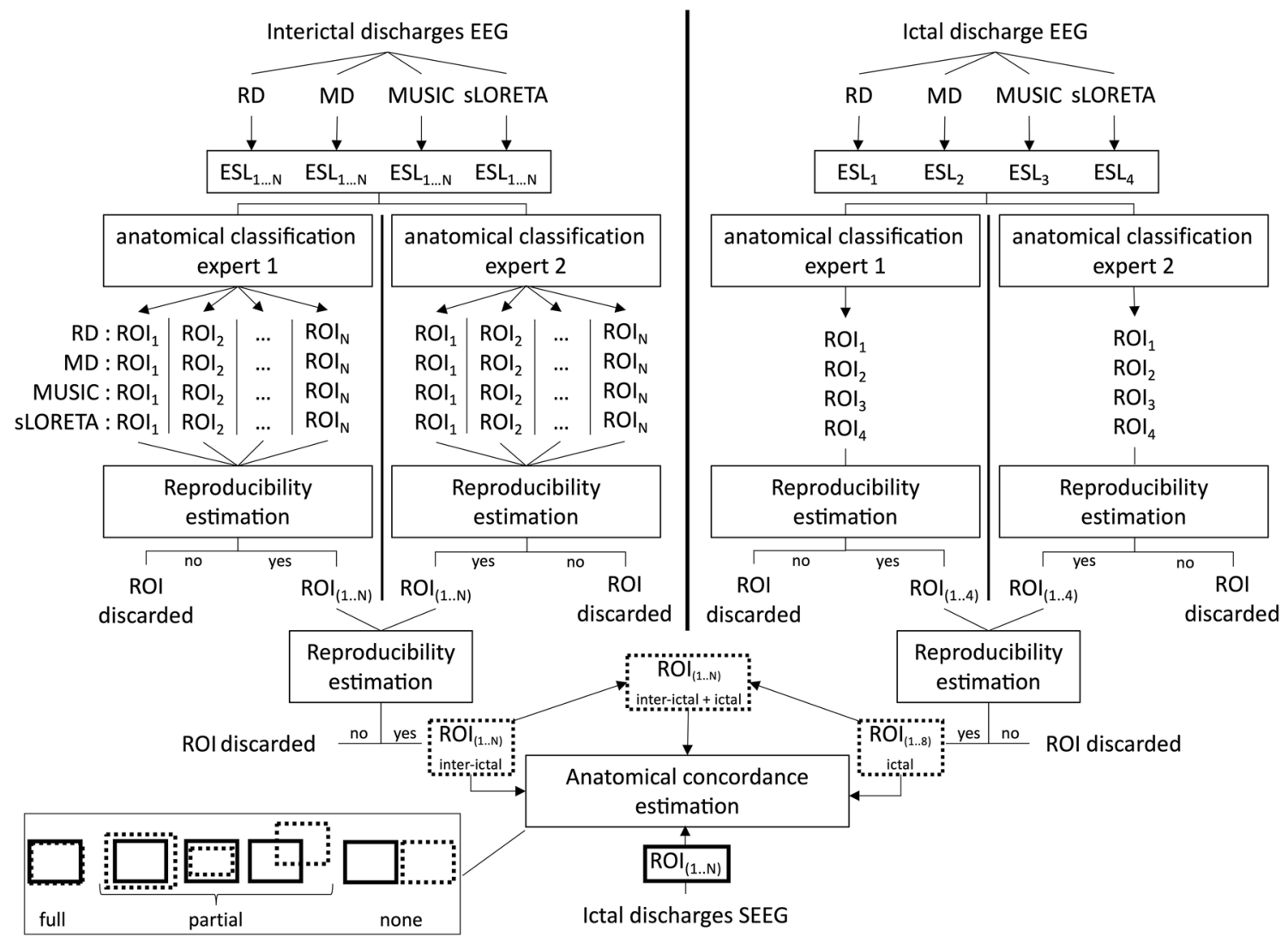

Fig. 1 Consecutive steps of electrical imaging analysis. Two different interpreters analyzed the electrical source localizations (ESL) prior to the SEEG investigation. Four different inverse problem methods were used (rotating dipole, moving dipole, MUSIC and sLORETA). Only reproducible ESL were selected and classified according to 36 predefined sub-lobar regions. At the end, we analyzed the anatomi-

Method (MUSIC) (Mosher et al. 1992 ) and a distributed source method (sLORETA) (Hämäläinen and Ilmoniemi 1994; Pascual-Marqui 2002).

ECD analysis was performed over the full duration of the selected temporal window with a moving dipole, involving the calculation of a new dipole localization, orientation and amplitude that best reproduces the measured electric field for each millisecond, as well as with a rotating dipole, involving the calculation of a unique dipole localization across the time window of analysis whereas orientation and amplitude were estimated for each millisecond. For IIDs, the localization of the moving dipole was considered optimal at the time point where goodness of fit (GOF), reflecting the percentage of EEG data explained by the model, was maximal, generally corresponding to the IID peak with maximal amplitude ratio. Stability of the source over the course of the IID ascending phase and peak was also assessed. For ictal discharges, the localization of the moving dipole was considered optimal when it remained stable in the same area for sufficient time (20-30 ms) and with a GOF $>50 \%$. No cal concordance (full, partial and none) between the sub-lobar regions obtained from electrical source imaging (interictal, ictal and the combination of both) and the sub-lobar regions from the stereoelectroencephalography investigation. ESL electrical source localization, ROI region of interest, $\mathrm{RD}$ rotating dipole, MD moving dipole

dipole confidence volume was measured but, as described by Fuchs et al. (2004), the size of the axes of the confidence ellipsoids is inversely proportional to the SNR of the measured data (Fuchs et al. 2004). Thus, the confidence volume is inversely proportional to the third power of the SNR. No noise floor regularization was done with ECD models. Noise was estimated by the SNR ratio.

We further applied a MUSIC method that uses a 3D dipole grid $(10 \mathrm{~mm})$ model placed in the brain volume combined with the principal component analysis (PCA) method (Mosher et al. 1992). The standardized low-resolution brain electromagnetic tomography (sLORETA) procedure relied on a distributed source model (Hämäläinen and Ilmoniemi 1994; Pascual-Marqui 2002) to provide a 3D activity distribution over time. For MUSIC, noise floor regularization was performed using the PCA decomposition on the time window of analysis and the selection of the eigenvectors (dipoles) which explain $95 \%$ of the signal subspace. For sLORETA, we used the interval window for which we computed the inverse solution to estimate the noise floor. Noise 
was assumed to be independent in each sensor and taken into account using regularization parameter. In our study, regularization was estimated via Generalized Cross Validation based on "leave one out" method (Pascual-Marqui 2002; Trujillo-Barreto et al. 2004).

\section{Determination of the Sub-lobar Localization of ESI Results}

Four experienced epileptologists (LGM, JPV in Nancy; MG, FB in Marseille) prospectively and independently interpreted the inter-ictal and ictal ESI results in the individual anatomical MRI for each patient.

For inter-ictal discharges, the anatomical localization of each source was obtained from the coordinates of moving and rotating dipoles with $\mathrm{GOF}>90 \%$, of the equivalent dipoles explaining more than $95 \%$ of the signal (eigenvector decomposition) using MUSIC, and of the dipoles with the highest magnitude using sLORETA (more details in Rikir et al. 2014).

For each selected ictal discharge, ESI was performed with the four above-mentioned source models. In case of discordance between source models, each localization was considered relevant. In those cases, this could lead to several sub-lobar localizations for a single ictal EEG discharge.

These anatomical localizations were then classified according to 18 predefined sub-lobar regions in each hemisphere: ventral medial prefrontal, dorsal medial prefrontal, ventral lateral prefrontal, dorsal lateral prefrontal, medial premotor, lateral premotor, medial central, lateral central, medial anterior temporal, lateral anterior temporal, medial posterior temporal, lateral posterior temporal, medial parietal, lateral parietal, medial occipital, lateral occipital, operculo-insular and temporo-parieto-occipital junction (Rikir et al. 2014). The size of each sub-lobar region was on average $34 \pm 18 \mathrm{cc}$ (min: $8 \mathrm{cc}$ for lateral premotor area and max: 68 cc for dorsal lateral prefrontal) (SPM Wake Forest University (WFU) Pickatlas toolbox and AAL templatehttps://www.fmri.wfubmc.edu/cms/software, version 3.05). In case of discordance between the two interpreters, further joint analysis led to consensus.

This step was performed several months prior to SEEG and therefore blinded to its results (Fig. 1).

\section{SEEG Recordings and Analysis}

Intra-cerebral depth electrodes (Nancy: Dixi Medical, Besançon, France; Marseille: Alcis, Besançon, France) consisting of 5-15 contiguous contacts (length $2 \mathrm{~mm}$, interval $1.5 \mathrm{~mm}$ ) were stereotactically placed under general anesthesia (Salado et al. 2018). A post-surgical CT-scan performed to rule out hemorrhage was fused with the pre-surgical MRI to precisely visualize depth electrode positions.

SEEG, combined with HD-video, was recorded $20 \mathrm{~h}$ a day for 5 to 7 days under the same conditions as 64-channel scalp EEG. Spontaneous and electrically induced SEEG seizures were analyzed by one of four experienced epileptologists (ER, LGM, JPV, MG) in order to estimate the EZ, defined as "the anatomical location of the site of the beginning and of the primary organization of the epileptic discharge" (Kahane et al. 2006). SEEG estimated EZ (SEEGEZ) was then classified according to the same 18 predefined sub-lobar regions applied to ictal and inter-ictal electrical source localization (ESL).

\section{Purposes}

\section{Ictal ESI Localizing Value and Subgroup Analysis}

The ictal ESL and SEEG-EZ sub-lobar concordance was assessed and classified as fully concordant, partly concordant, or discordant for each patient. Full concordance corresponded to a complete matching between ictal ESL and EZ sub-lobar localizations (iESL=EZ). Partial concordance corresponded to a partial matching between ictal ESL and EZ sub-lobar localizations and encompassed three different conditions: (i) ESL pointed to the full EZ as well as to additional sub-lobar localizations (iESL $>$ EZ); (ii) ESL pointed only to some EZ sub-lobar localizations (iESL $<$ EZ); (iii) ESL pointed to some EZ sub-lobar localizations and to additional localizations outside the EZ (iESL $><$ EZ). ESL and EZ were discordant if they had no common sub-lobar localization (iESL $\neq$ EZ) (Fig. 1) (Rikir et al. 2014).

We defined ictal ESI sensitivity as the percentage of patients with full or partial concordance between ictal ESL and EZ, i.e. the probability to localize at least part of the EZ with ictal ESI. Ictal ESI specificity was defined as the percentage of patients with ictal ESL fully concordant with or entirely contained in the EZ i.e. the probability of not localizing with ESI outside the EZ (the probability of ictal ESI to localize strictly within the EZ) (Rikir et al. 2017).

Ictal ESI sensitivity and specificity were first assessed for the whole cohort and then in subgroups according to:

(a) The morphology of the ictal discharge (IS, RD $>13 \mathrm{~Hz}$ and $\mathrm{RD}<13 \mathrm{~Hz}$ );

(b) The presence of a structural lesion on MRI (positive MRI vs negative MRI);

(c) The presumed etiology. For operated patients, etiology was determined based on the pathological findings, while for non-operated ones, it was determined based on the combination of MRI and/or electrophysiological findings. All cases were classified in three categories: 
(i) malformations of cortical development (MCD), (ii) others including atrophy, porencephaly, hippocampal sclerosis (HS) and (iii) unknown for non-operated patients without MRI lesion and without typical SEEG pattern suggestive of MCD (Chassoux et al. 2000).

\section{Inter-ictal ESI Localizing Value}

We further assessed the sub-lobar concordance of inter-ictal ESL (iiESL) with SEEG estimated EZ according to the same definitions as for ictal ESL. Inter-ictal ESI sensitivity and specificity were assessed according to the same definitions as for ictal ESI. Results were then analyzed according to (a) the presence of a structural lesion on MRI (positive or negative) and (b) the presumed etiology as for ictal ESI (Fig. 1).

\section{Comparison of Ictal and Inter-ictal ESI Localizing Value}

Ictal and inter-ictal ESI sensitivity and specificity were compared for the whole cohort and according to the abovementioned axis. We assessed the added value of ictal ESI and inter-ictal ESI (Fig. 1).

Ictal ESI was considered to offer an added value, validated by SEEG, in cases of:

(a) Discordance between iiESL and SEEG-EZ but partial or perfect concordance between iESL and SEEG-EZ.

(b) Partial concordance with iiESI (iiESL $><$ SEEG-EZ or iiESL $>$ SEEG-EZ) but partial (iESL $<$ SEEG-EZ) or perfect concordance (iESL $=$ SEEG-EZ) with iESI.

Inter-ictal ESI was considered to offer an added value, validated by SEEG, in cases of:

(a) Discordance between iESL and SEEG-EZ but partial or perfect concordance between iiESL and SEEG-EZ

(b) Partial concordance with iESI (iESL $><$ SEEG-EZ or iESL $>$ SEEG-EZ) but partial (iiESL $<$ SEEG-EZ) or perfect concordance (iiESL $=$ SEEG-EZ) with iiESI.

We also assessed the anatomical concordances of ictal plus inter-ictal ESL with SEEG estimated EZ by combining, for each patient, all sub-lobar regions found for interictal and ictal analysis (Fig. 1). Finally, we calculated the sensitivity and specificity of the combined ictal plus inter-ictal ESI $(\mathrm{i}+\mathrm{ii} \mathrm{ESI})$.

Statistical comparisons between ictal and inter ictal ESI sensitivity and specificity were performed using McNemar Test (Biostat TGV, Jussieu University) for the whole cohort. The following $H O$ hypothesis was tested: the nature of the EEG signal (ictal or inter-ictal) did not influence sub-lobar concordance between ESL and SEEG-EZ. Values of $p \leq 0.05$ were considered significant. A global statistical analysis was also performed across all subgroups (regarded as variables) using generalized linear models (GLM) (Jamovi software, version 1.2; https://www.jamovi.org). We tested whether the ictal ESL concordance with SEEG-EZ was dependent on one or several of our subgroups (MR lesion or not, etiology and ictal patterns) and on the inter-ictal ESL concordance. We also performed the same test for the inter-ictal ESL concordance and ictal plus inter-ictal ESL concordance with SEEG-EZ.

\section{Results}

\section{Patients}

We prospectively enrolled 78 patients (38 women) with mean age 31 years at inclusion. Twenty-four patients were excluded from the analysis because of failure to record seizure during 64ch scalp VIDEO-EEG in 17, eventually not performed SEEG in 5, and inconclusive SEEG in 2.

Among the 54 analyzed patients, mean age at inclusion was $30.3 \pm 8.8$ years; mean epilepsy duration was $18.6 \pm 9.5$ years. One to thirty seizures were recorded per patient during $64 \mathrm{ch}$ scalp video-EEG recordings (mean $5.7 \pm 6.4$; median 3 ).

Twenty-four of 54 (44\%) patients had temporal lobe epilepsy (TLE) and 30 (56\%) had extra-temporal epilepsy (ETLE): 10 (19\%) frontal lobe epilepsy (FLE), six (11\%) posterior epilepsy (arising from the occipital or parietal region), four (7\%) operculo-insular epilepsy and 10 (19\%) multi-lobar epilepsy. MRI was negative in $17(31 \%)$ patients and showed a lesion in $37(69 \%)$.

Thirty-tree patients (61\%) had a MCD: 16 focal cortical dysplasia (FCD), six dysembryoplasic neuroepithelial tumor (DNT), three polymicrogyria (PMG), two ganglioglioma, two neuronal heterotopy, one Tuberous Sclerosis Complex (TSC) and three had no lesion on MRI but typical electric pattern on SEEG suggestive of MCD (Chassoux et al. 2000). Five $(9 \%)$ patients had gliosis, three (5\%) cortical atrophy, nine (17\%) HS (Table 1).

\section{Ictal EEG Pattern Characteristics}

One representative seizure per patient was selected except for patient 6 and patient 20 who had two distinct types of seizure. For both, one seizure of each type was selected leading to an analysis of 56 seizures among 54 patients.

Fifty (89\%) of 56 ictal EEG events retained for ESI occurred before the first objective ictal clinical sign with a mean electro-clinical delay of $6.1 \mathrm{~s}$.

Forty-five (80\%) of 56 analyzed ictal EEG events were the first identifiable ictal EEG pattern. In the 11 remainders, 


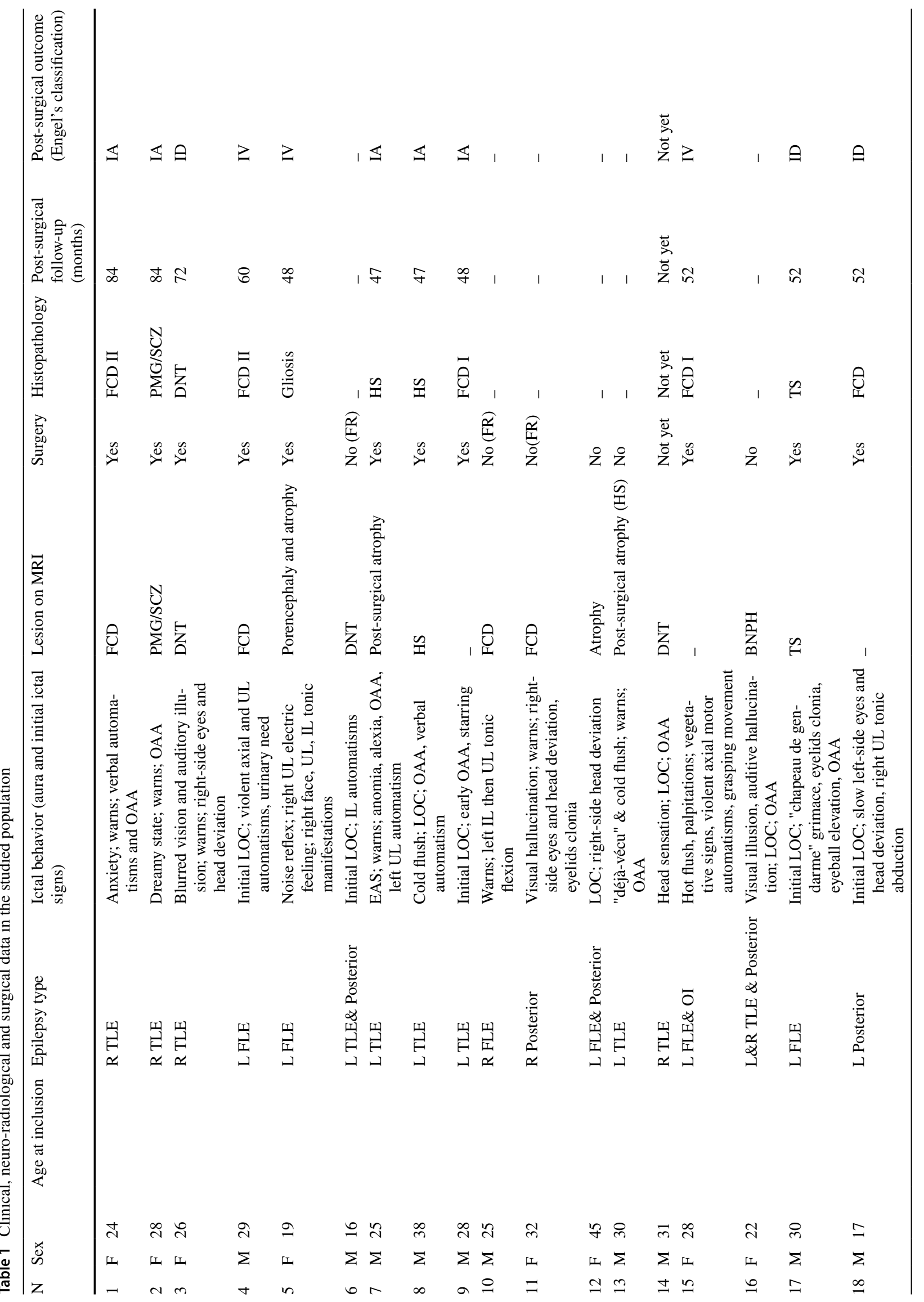




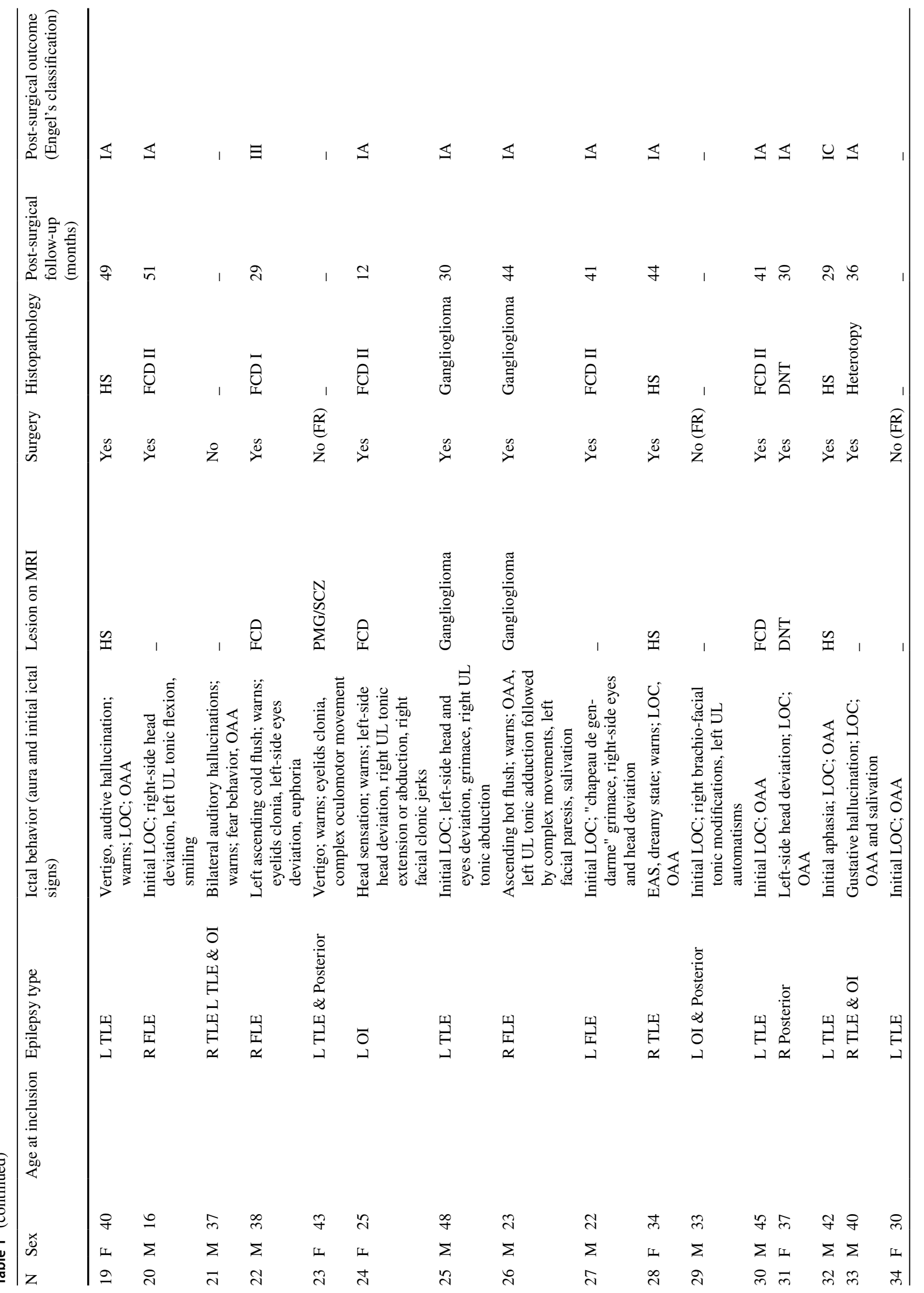




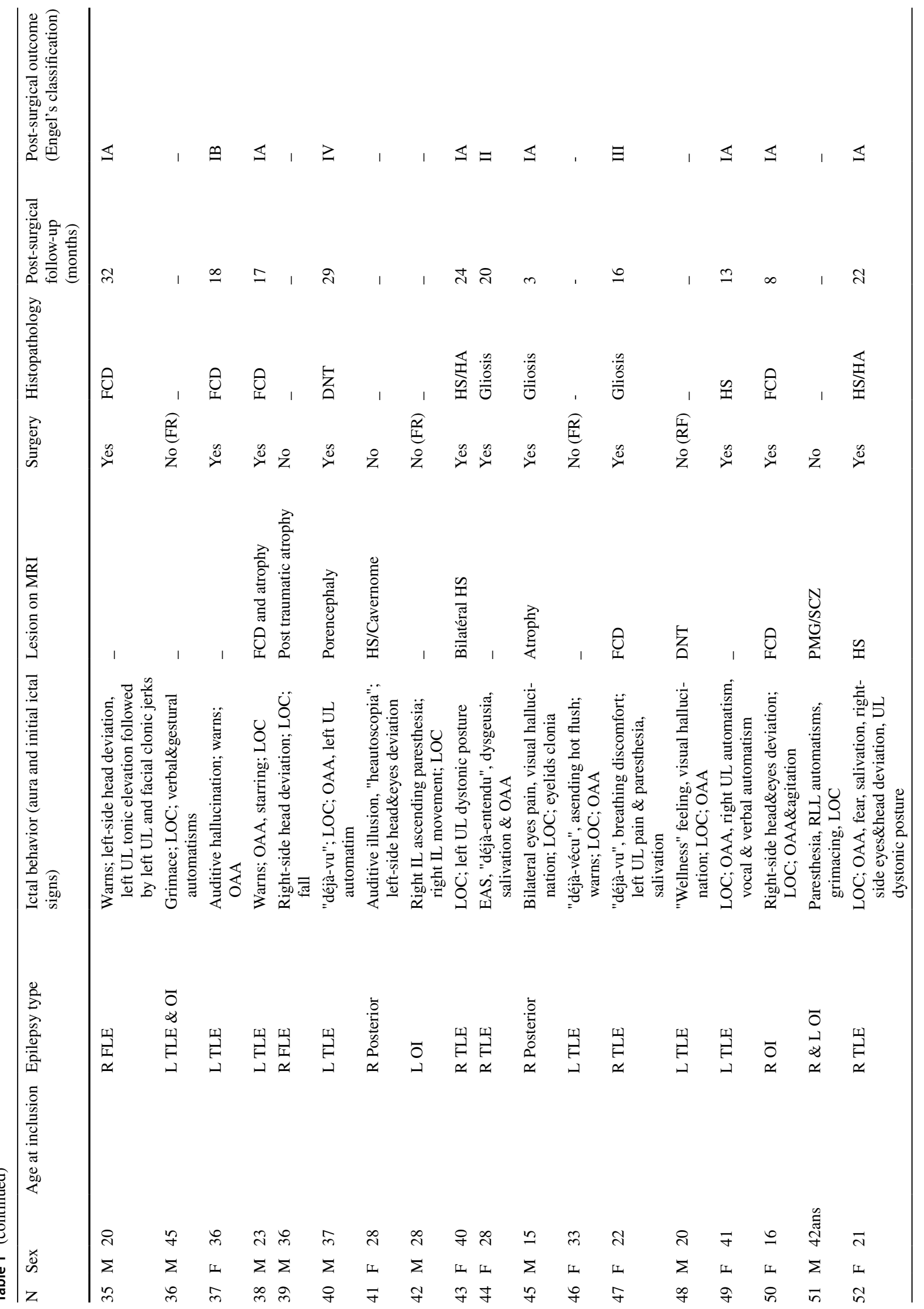




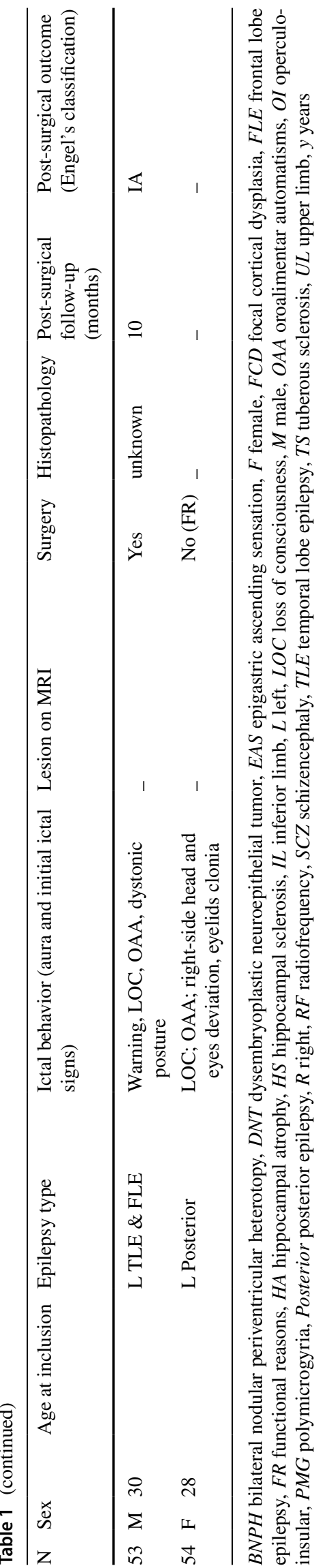

the initial ictal event was a regional flattening and the first subsequent ictal rhythmic discharge was analyzed (temporal delay from 2 to $21 \mathrm{~s}$, mean $11.45 \mathrm{~s}$ ).

Analyzed ictal EEG events were IS in 29 cases (52\%), $\mathrm{RD}<13 \mathrm{~Hz}$ in 19 (34\%) and $\mathrm{RD}>13 \mathrm{~Hz}$ in eight (14\%).

\section{Ictal ESI Results}

Ictal ESL and EZ were fully concordant in $12(21 \%)$ cases, partly concordant in 30 (54\%), and discordant in 14 (25\%). Among the partly concordant cases: (i) iESL was entirely included into the EZ in 16 cases (iESL $<$ EZ); (ii) iESL entirely included the EZ in 8 cases (iESL $>$ EZ); (iii) iESL and EZ partially overlapped in 6 cases (iESL $><$ EZ).

When the ictal EEG pattern was an IS $(n=29)$, iESL was fully concordant with EZ in 8 (28\%), partly concordant in $14(48 \%)$ and discordant in 7 (24\%). In the RD> $13 \mathrm{~Hz}$ subgroup $(n=8)$, iESL was fully concordant with EZ in 2 (25\%), partly concordant in $3(38 \%)$ and discordant in 3 $(38 \%)$ whereas in the $\mathrm{RD}<13 \mathrm{~Hz}$ subgroup $(\mathrm{n}=19)$, concordances were respectively $11 \%, 68 \%$ and $21 \%$.

In case of negative MRI $(n=19)$, iESL was fully concordant with EZ in 5 (26\%), partly concordant in $10(53 \%)$ and discordant in $4(21 \%)$. When MRI showed a structural lesion $(n=37)$, iESL was fully concordant with EZ in $7(19 \%)$, partly concordant in 20 (54\%) and discordant in 10 (27\%).

In case of MCD $(n=35)$, iESL was fully concordant with EZ in 10 (29\%), partly concordant in $18(51 \%)$ and discordant in $7(20 \%)$ whereas in "others" subgroup $(n=17)$, including $9 \mathrm{HS}, 5$ gliosis and 3 atrophy, concordances were respectively $12 \%, 53 \%$ and $35 \%$ (Table 2 ).

According to the GLM statistical test, ictal ESL concordances with EZ-SEEG were not dependent on the MRI $(\mathrm{p}=0.42)$ and the ictal EEG pattern $(\mathrm{p}=0.43)$ subgroups but dependent on the etiology subgroup $(\mathrm{p}=0.04)$ and on the interictal ESL concordances with EZ-SEEG $(\mathrm{p}<0.001)$.

Ictal ESI sensitivity and specificity amounted to $75 \%$ and $50 \%$ in the whole cohort.

\section{Inter-ictal ESI Results}

Inter-ictal ESL and EZ were fully concordant in $10(18 \%)$, partly concordant in 37 (66\%), and discordant in 9 patients (16\%). Among the partly concordant cases: (i) iiESL was entirely included into the EZ in 11 (iiESL $<$ EZ); (ii) iiESL entirely included the EZ in 9 (iiESL $>$ EZ); (iii) iiESL and EZ partially overlapped in 17 patients (iiESL $><$ EZ).

In case of negative MRI $(n=19)$, iiESL was fully concordant with EZ in 5 (26\%), partly concordant in $12(63 \%)$ and discordant in two (11\%). When MRI showed a structural lesion $(\mathrm{n}=37)$, iiESL was fully concordant with EZ in 5 (14\%), partly concordant in $25(67 \%)$ and discordant in 7 $(19 \%)$. 


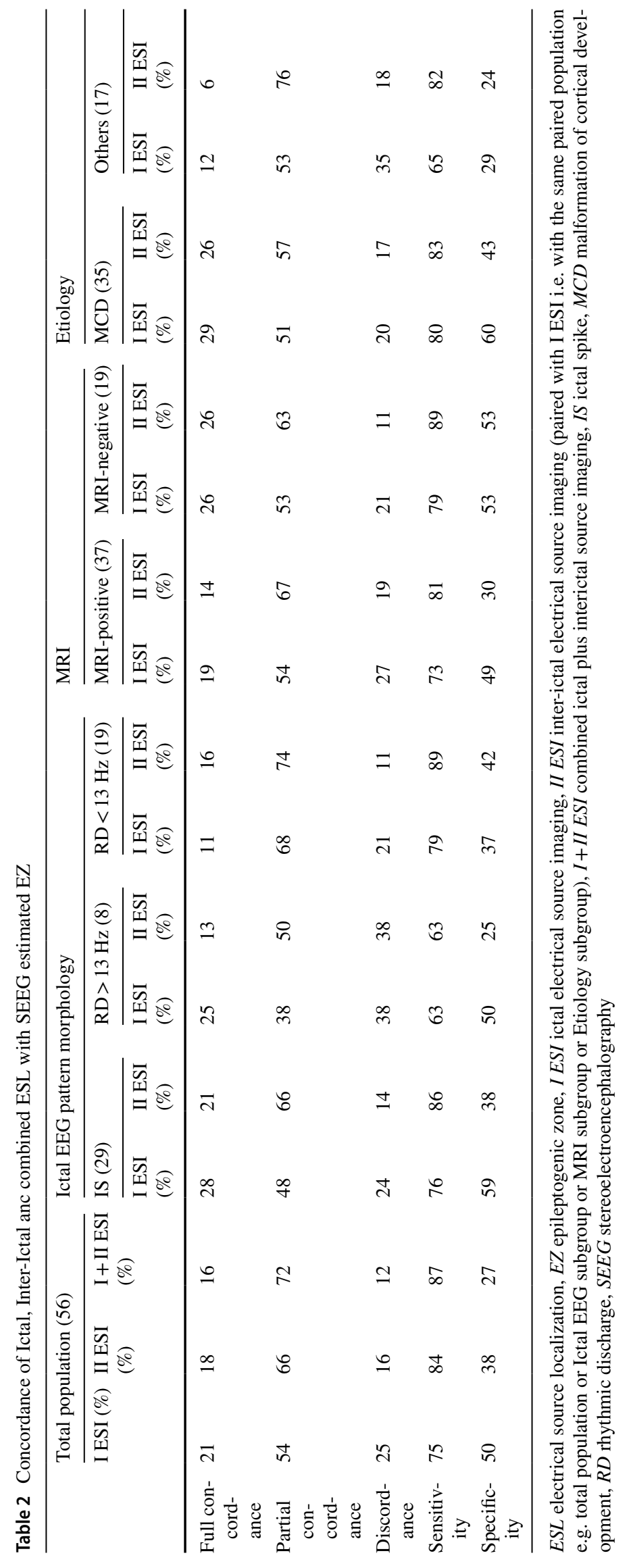


In case of MCD $(n=35)$, iiESL was fully concordant with EZ in $9(26 \%)$, partly concordant in $20(57 \%)$, and discordant in $6(17 \%)$ whereas in case of other etiologies $(\mathrm{n}=17)$, concordances were respectively $6 \%, 76 \%$, and $18 \%$ (Table 2).

According to the GLM statistical test, interictal ESL concordances with EZ-SEEG were not dependent on the MRI $(p=0.62)$ and the etiology $(p=0.33)$ subgroups but dependent to ictal ESI concordances with EZ-SEEG $(\mathrm{p}<0.001)$.

Inter-ictal ESI sensitivity and specificity amounted to $84 \%$ and $38 \%$ in the whole cohort.

\section{Ictal and Inter-ictal ESI Comparison}

Inter-ictal and ictal ESI sensitivity ( $84 \%$ and $75 \%$ respectively) and specificity (38\% and 50\% respectively) were similar in the whole cohort (McNemar Test, $\mathrm{p}=0.18$ ). GLM tests demonstrated that the ictal and interictal ESL concordances with SEEG-EZ were interdependent $(\mathrm{p}<0.001)$.

Whatever the subgroup, the inter-ictal ESI sensitivity was always higher than ictal ESI.

Subgroup analysis showed a higher specificity for ictal compared to inter-ictal ESI in RD $>13 \mathrm{~Hz}(50 \%$ vs $25 \%)$ and in ictal spike subgroups (59\% vs 38\%), in MCD (60\% vs $43 \%$ ) and in MRI positive patients (49\% vs $30 \%$ ).

Ictal ESI improved the EZ localization compared to iiESI in 14 cases (25\%): (i) two cases showed discordance between iiESL and SEEG-EZ but showed a concordance between iESL and SEEG-EZ (Figs. 2, 3) and (ii) 12 cases were partially concordant on inter-ictal analysis (iiESL $><$ EZ or iiESL $>$ EZ) and fully concordant (iESL $=$ EZ) or entirely included into the EZ (iESL $<$ EZ) on ictal analysis (Figs. 4, 5). Among these 14 cases, 10 (71\%) had a MCD and 10 (71\%) had a structural MRI lesion.

Inter-ictal ESI improved the EZ localization compared to iESI in 13 cases (23\%): (i) 7 cases showed discordance between iESL and SEEG-EZ but showed a concordance between iiESL and SEEG-EZ and (ii) 6 cases were partially concordant on ictal analysis (iESL $><$ EZ or iESL $>$ EZ) and were fully concordant (iiESL $=\mathrm{EZ}$ ) or entirely included into the EZ (iiESL $<$ EZ) on inter-ictal analysis. Among these 13 cases, 7 (54\%) had a no structural lesion on MRI and 6 (46\%) had a MCD.

Combination of ictal plus inter-ictal ESL yielded a sensitivity of $87 \%$ and a specificity of $27 \%$. In terms of concordance, combination of iESI plus iiESI decreased the perfect concordance (16\% instead of $21 \%$ with iESI and $17 \%$ with iiESI) but also decreased the discordance rate (12\% instead of $25 \%$ in iESI and $16 \%$ in iiESI).

Concordance of combined ictal plus inter-ictal ESL with SEEG-EZ was not dependent on any of the studied variables $(\mathrm{p}>0.625)$.

\section{Discussion}

The purposes of this study were to prospectively evaluate and compare the localizing value of ictal ESI and interictal ESI in the same subjects using SEEG as reference method for EZ definition. The strengths of our study entail (i) the prospective design warranting an interpretation of ESI blinded to the results of the SEEG, (ii) the application of a strictly uniform ESI methodology with long term 64-channel EEG recordings and realistic individual head models in a large cohort of focal refractory epileptic patients and (iii) the use of SEEG to delineate the EZ instead of the surgical resection volume that is often larger than the necessary minimum volume to be resected to obtain seizure freedom. Concerning this last point, we believe that electrophysiological definition of the EZ was physiologically more meaningful because: (1) it allows identifying not only the area of seizure initiation but also the areas of seizure propagation; (2) it includes all patients even those with surgical contraindication for functional reasons (Rikir et al. 2014).

Inter-ictal and ictal ESI sensitivity (84\% and 75\% respectively) and specificity (38\% and 50\% respectively) were in the same range in the whole cohort. A first superficial interpretation of these findings could be that extending the duration of high-resolution EEG recordings in order to perform ESL of ictal discharge is unhelpful to localize at least part of the EZ. However, our results suggest that ictal ESI increases the specificity of ESL that is the probability of not localizing the epileptic sources outside the EZ. This is consistent with a recent SEEG study (Bartolomei et al. 2016) demonstrating that the irritative zone is larger than the EZ. The lower specificity of inter-ictal ESI would result from the localization of the so-called secondary irritative zone broadly corresponding to the area of seizure propagation. Ictal ESI sensitivity was never higher than inter-ictal ESI among all subgroups. In Lascano et al. (2016), inter-ictal ESI sensitivity was in the same range $(87.8 \%)$ and the combination with other methods always decreased the sensitivity value (iiESI + MRI: $58.5 \%$; iiESI + PET: $58.5 \%$; iiESI + SPECT: 43.9\%). In our study, the combined analysis of ictal and interictal ESL did not change the value of sensitivity but substantially decreased the value of specificity. This indicates the relevance to perform separate analysis of ictal and interictal discharges and then to interpret both ESL results in a same patient.

From a clinical point of view, it seems interesting to identify which patients should benefit from ictal ESI in pre-surgical workup. Ictal ESI seemed to have a better specificity than inter-ictal ESI in cases of MCD $(60 \%$ vs $43 \%$ ) and in MRI positive patients (49\% vs $30 \%)$. Ictal ESI also improved the EZ localization compared to iiESI 
Fig. 2 Inter-ictal and ictal EEGSEEG patterns in a situation of full concordance between ictal ESI and SEEG but discordance between inter-ictal ESI and ictal ESI (patient 42). a Selected electrodes of interest from scalp 64-channel EEG recordings showing one type of IID in channels F7, FT7, T7, FT9, F5 and FC5 (monopolar montage, common average reference). b Selected electrodes of interest from ictal 64-channel scalp EEG recording. The seizure starts with a diffuse RD $>13 \mathrm{~Hz}$ but predominating in channels F7, FT7, T7, TP7, P7, PO7, FT9 and $\mathrm{P} 9$. The ictal semiology starts $1 \mathrm{~s}$ later (vertical arrow). c SEEG recordings showed ictal discharge occurring first in the left posterior insula and less predominantly in the middle and anterior insular cortex. (EZ epileptogenic zone, IID interictal discharge, $R D>13 \mathrm{~Hz}$ rhythmic discharge $>13 \mathrm{~Hz}$, SEEG stereoelectroencephalography)
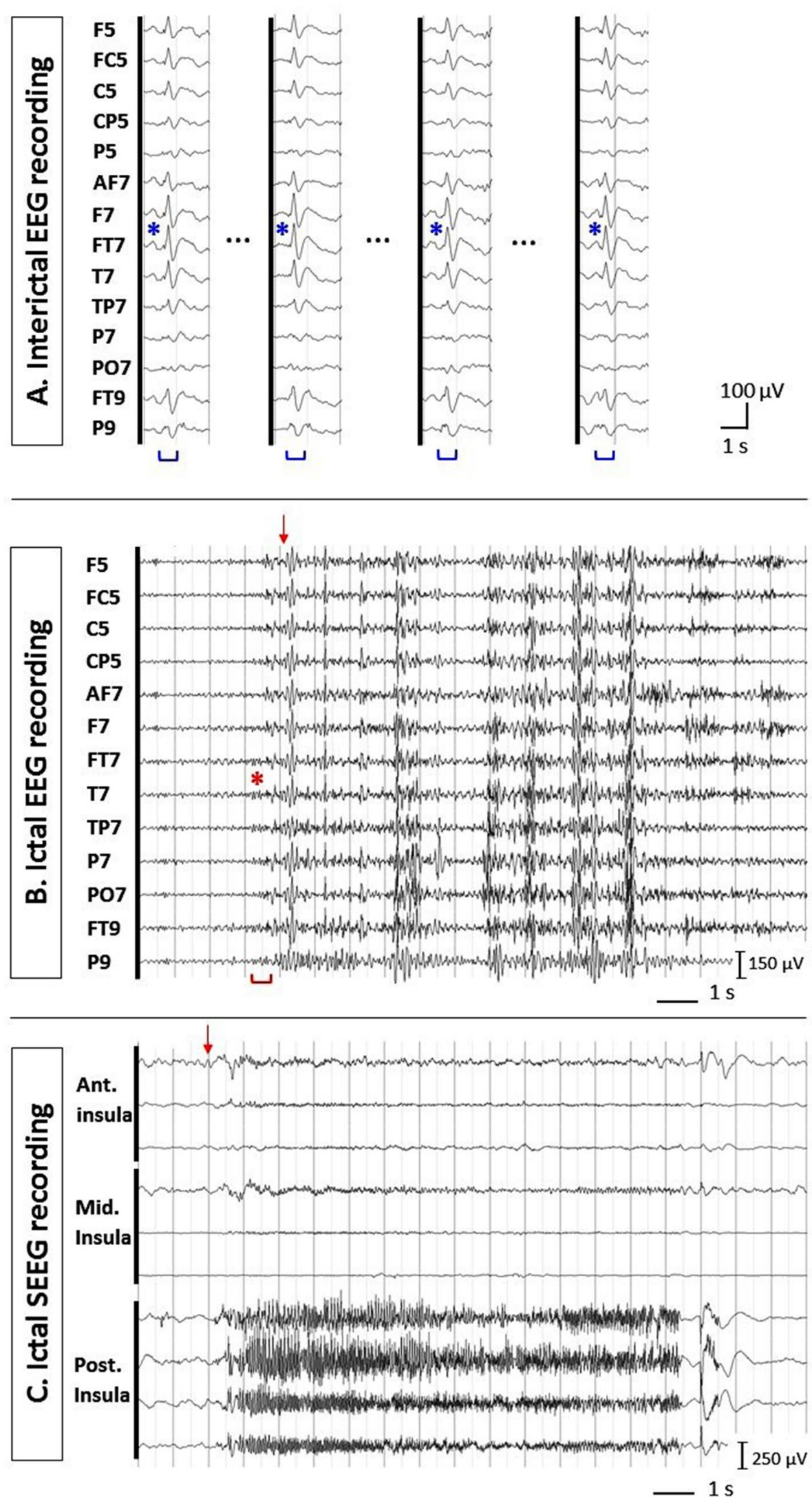


\section{Sublobar classifications}
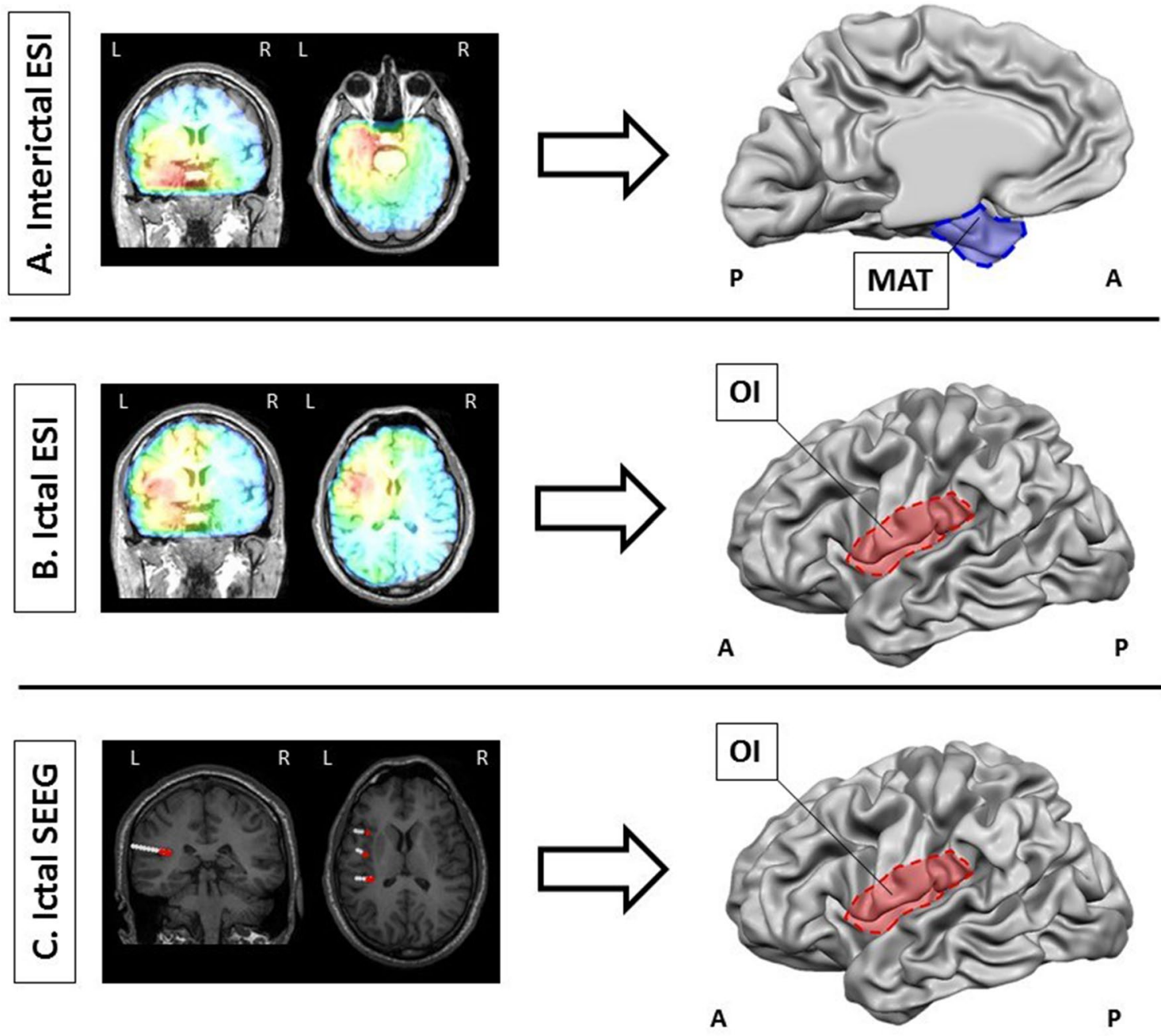

Fig. 3 Electrical source localizations and sub-lobar concordance analysis in a situation of full concordance between ictal ESI and SEEG but discordance between inter-ictal ESI and ictal ESI (patient 42). a Distributed source localization of IID (see Fig. 1a) in the anterior and mesial part of the left temporal pole (sLORETA). These anatomical localizations corresponded to a MAT sub-lobar classification. b Distributed source localization of ictal RD $>13 \mathrm{~Hz}$ (see Fig. 1b) in the left anterior insular cortex. This anatomical localization corresponded to an operculo-insular sub-lobar classification. c CT-MRI co-registration of 3 out of 14 depth electrodes. The most anterior depth electrode recorded the left anterior insula (internal contacts) and the prefrontal operculum (external contacts). The intermediary depth electrode recorded the left middle insula (internal contacts) and the

in 14 cases $(25 \%)$ of which $10(71 \%)$ had a MCD and 10 (71\%) had a structural MRI lesion. Our study suggests a greater relative benefit for ictal analysis in patients with (i) malformations of cortical development which constitute one of the main causes of neocortical refractory epilepsy (Spencer and Huh 2008; Papayannis et al. 2012) or (ii) a lesion on MRI. In contrast, ictal analysis seemed to present central operculum (external contacts). The most posterior electrode recorded the left posterior insula (internal contacts) and the parietal operculum (external contacts). Red contacts are those involved in EZ. The sub-lobar classification of EZ was consequently operculoinsular. Considering the sub-lobar classification column, iiESL was discordant with SEEG-EZ while iESL was fully concordant with SEEG-EZ illustrating the potential added value of ictal ESI to interictal ESI (ESI electrical source imaging, EZ epileptogenic zone, iESL ictal electrical source localization, iiESL inter-ictal electrical source localization, IID inter-ictal discharge, MAT mesial anterior temporal, OI operculo-insular, RD $>13 \mathrm{~Hz}$ rhythmic discharge $>13 \mathrm{~Hz}$, SEEG stereoelectroencephalography, sLORETA standardized low-resolution brain electromagnetic tomography)

no benefit compared to inter-ictal analysis in MRI negative patients (iESI and iiESI sensitivity: $79 \%$ and $89 \%$; iESI and iiESI specificity: 53\% and 53\%). This might be partly explained by the higher overlap between irritative and epileptogenic zones in the main etiological substrate of this subgroup (11/19 FCD) (Bartolomei et al. 2016; Chassoux et al. 2000). 
Fig. 4 Inter-ictal and ictal EEGSEEG patterns in a situation of full concordance between ictal ESI and SEEG but partial concordance between inter-ictal ESI and ictal ESI (patient 19). a Selected electrodes of interest from scalp 64-channel EEG recordings showing 2 types of IID. First IID type in channels F1, FC1, F3, FC3, F5, FC5 and $\mathrm{F} 7$ and second one in channels T3, TP7, CP5 (monopolar montage, common average reference). b Selected electrodes of interest from ictal 64-channel scalp EEG recording. The seizure starts with ictal spikes in channels F3, FC3, F5, FC5, F7, FT7 followed by RD $<13 \mathrm{~Hz}$.

The ictal semiology starts $1 \mathrm{~s}$ later (vertical arrow). c SEEG recordings showed ictal discharge occurring first in the left amygdala and the anterior entorhinal cortex (IID inter-ictal discharge, $\mathrm{RD}<13 \mathrm{~Hz}$ rhythmic discharge $<13 \mathrm{~Hz}$, SEEG stereo-electroencephalography)
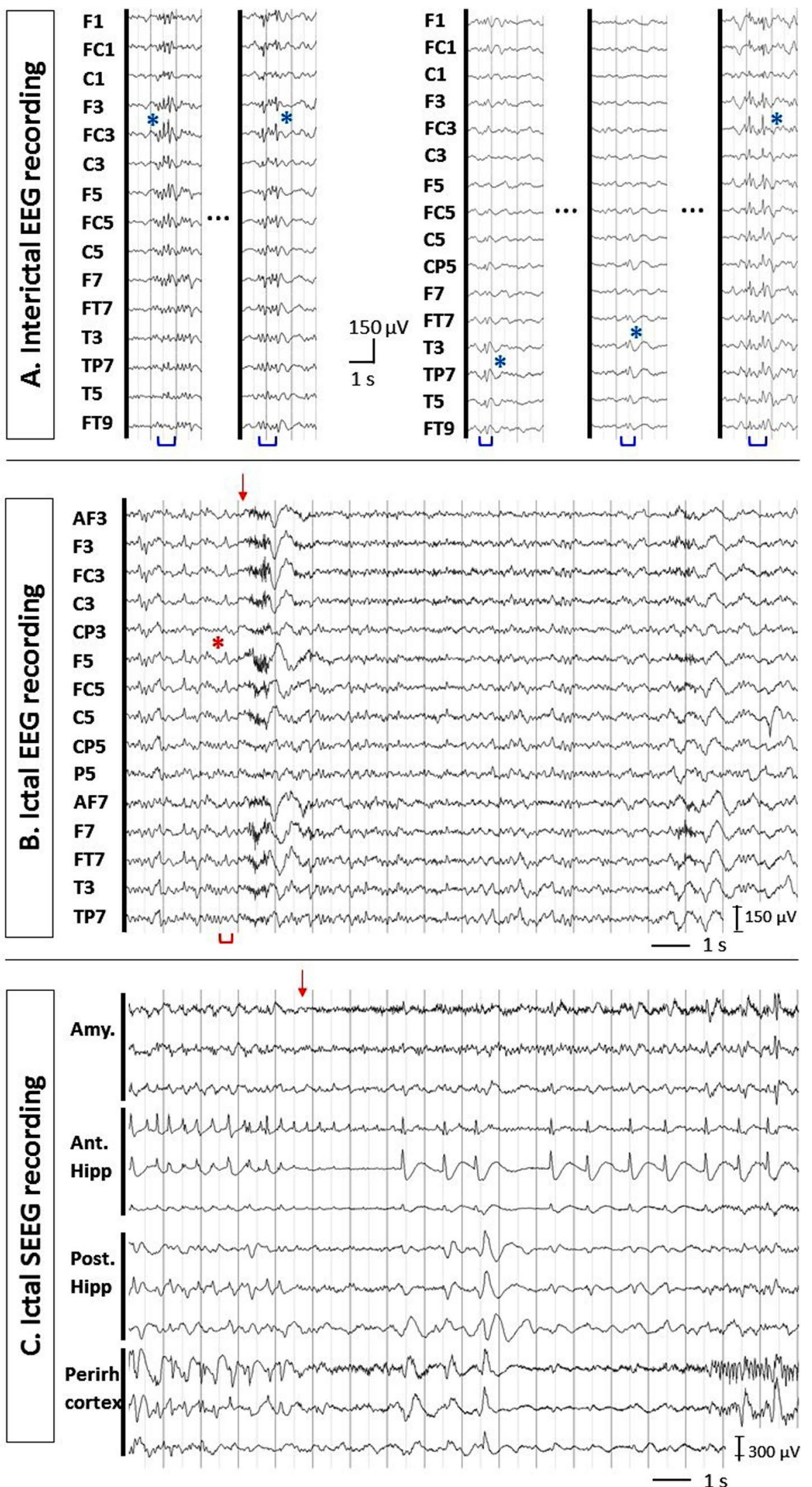

$1 \mathrm{~s}$ 

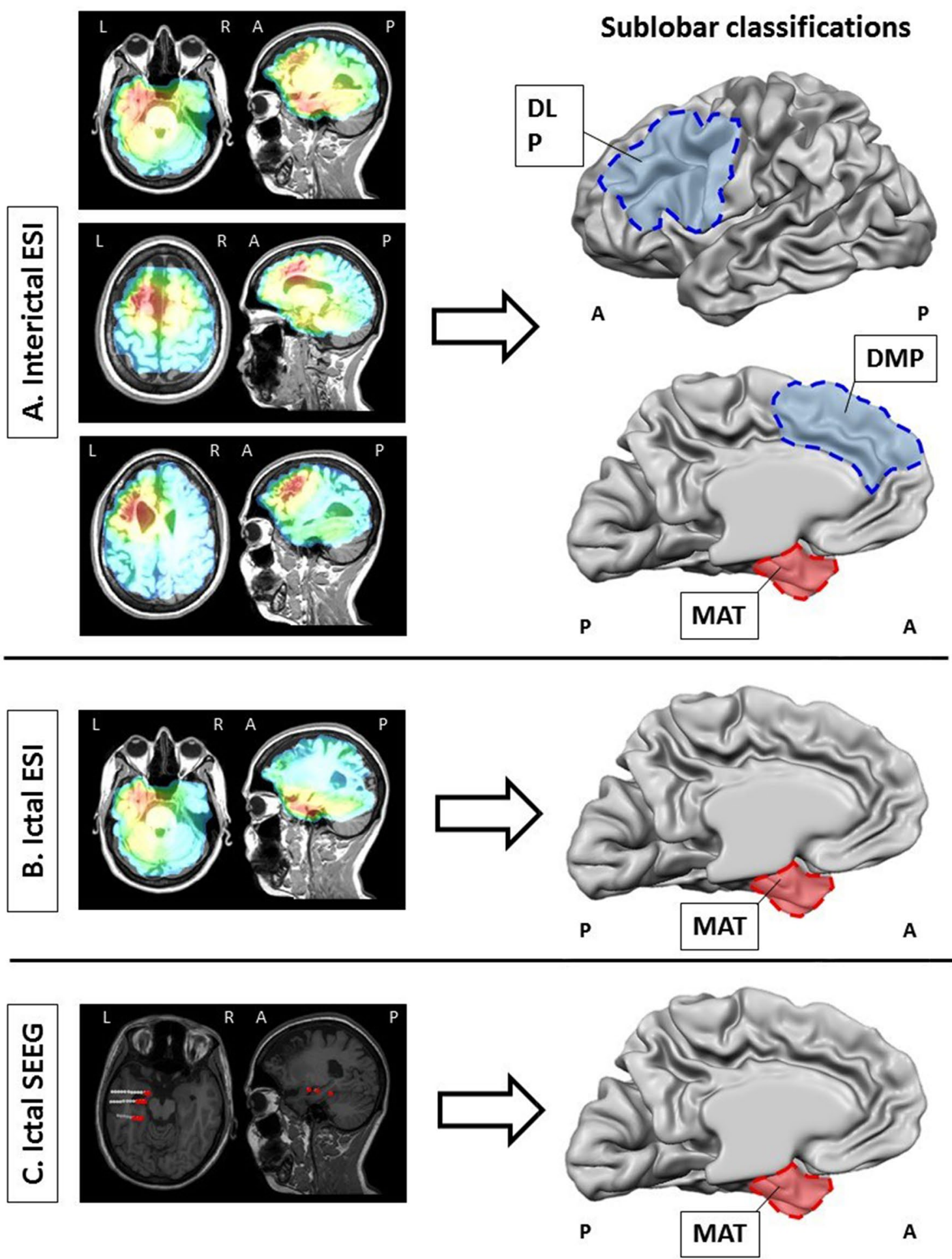

How could all MCD patients benefit from an ictal analysis although FCD patients could not? FCD represented $57 \%$ of our MCD subgroup (20 FCD and 15 non FCD patients). Difference between iiESI and iESI specificity was greater for the non FCD patients (iiESI and iESI specificity: $40 \%$ and $50 \%$ for FCD; $47 \%$ and $67 \%$ for non FCD). Ictal ESI improved EZ localization compared to iiESI in $30 \%$ FCD patients (6/20) and in 53\% non FCD patients (8/15). Again, this might be partly explained by the more heterogenous etiological substrates in non FCD patients (PMGs, glioneural tumors, and tubers) in which the irritative zone rarely perfectly overlaps and may be more often discordant with 
४Fig. 5 Electrical source localizations and sub-lobar concordance analysis in a situation of full concordance between ictal ESI and SEEG but partial concordance between inter-ictal ESI and ictal ESI (patient 19). a Distributed source localization of IID (see Fig. 3a) in the anterior part of MFG and mesial part of SFG (type 1) and in the anterior and mesial part of the left temporal pole (type2) (sLORETA). These anatomical localizations corresponded to DLPref; DMPref and MAT sub-lobar classification. b Distributed source localization of ictal spike (see Fig. 3b) in the left anterior and mesial part of temporal pole. This anatomical localization corresponded to a MAT sublobar classification. c CT-MRI co-registration of 3 out of 14 depth electrodes. The most anterior depth electrode recorded the left amygdala (internal contacts) and the left MTG (external contacts). The intermediary depth electrode recorded the left anterior hippocampus (internal contacts) and the left MTG (external contacts). The most posterior electrode recorded the left parahippocampal gyrus (internal contacts) and the ITG (external contacts). Red contacts are those involved in EZ. The sub-lobar classification of EZ was consequently MAT. Considering the sub-lobar classification column, iiESL was partly concordant with SEEG-EZ (iiESL > SEEG-EZ) while iESL allowed a restriction of localization that was validated by SEEG. This case illustrates the potential added-value of ictal ESI to inter-ictal ESI (DLPref dorsal lateral prefrontal, DMPref dorsal mesial prefrontal, ESI electrical source imaging, $E Z$ epileptogenic zone, $i E S L$ ictal electrical source localization, IID inter-ictal discharge, iiESL inter-ictal electrical source localization, ITG inferior temporal gyrus, $M A T$ mesial anterior temporal, $M F G$ middle frontal gyrus, $M T G$ middle temporal gyrus, $O I$ operculo-insular, $R D>13 \mathrm{~Hz}$ rhythmic discharge $>13 \mathrm{~Hz}, S E E G$ stereo-electroencephalography, $S F G$ superior frontal gyrus, sLORETA standardized low-resolution brain electromagnetic tomography)

the epileptogenic zone (Maillard et al. 2009; Ramantani et al. 2013). Moving from an inter-ictal to an ictal analysis in these cases decreases the probability of localizing a region outside the EZ.

When ictal ESI is considered, concordance seems related to the morphology of the ictal EEG discharge: the sensitivity for IS and $\mathrm{RD}<13 \mathrm{~Hz}$ being higher than for $\mathrm{RD}>13 \mathrm{~Hz}$. RD $>13 \mathrm{~Hz}$ have usually a low SNR $(<2)$ that could partly explain this result. However, iESI specificity is better for IS and RD $>13 \mathrm{~Hz}$ subgroups (59 and 50\%) compared to $\mathrm{RD}<13 \mathrm{~Hz}$ subgroup (37\%). IS and RD $>13 \mathrm{~Hz}$ were the first visible ictal EEG modification in $100 \%$ and $87.5 \%$ of cases respectively whereas $\mathrm{RD}<13 \mathrm{~Hz}$ was the first visible ictal discharge in only $47 \%$. This suggests that the earlier the scalp ictal EEG discharge is, the more specific of the EZ the source localization is. In other words, localizing from early ictal EEG event increases the probability of not localizing outside the EZ. This is in accordance with intracerebral studies that demonstrated that $\mathrm{RD}<13 \mathrm{~Hz}$ is a typical pattern of propagation occurring at the end of seizures (Lagarde et al. 2016).

\section{Limits}

Some limitations are related to our study design. Due to the prospective design of the study, scalp and intra-cerebral EEG recordings were not simultaneous. The scalp seizure selected for ESI might not exactly have the same clinical course than those used during SEEG recordings to assess the EZ localization. However, we checked this issue « a posteriori» and found no major discrepancies. This methodological issue is inherent to the technic and common to all previous cohort ESL studies. In this study, we choose to analyze the ictal patterns in the temporal domain in order to see if only ESI (i.e. without pre-processing signal analysis) can localize precisely the EZ. A way to improve the precision of the source localization, especially for the RD analysis, could be to perform the ESI in the frequency domain. Several studies demonstrated the promising aspect of this method especially in case of low SNR and correlated sources (Lantz et al. 1999; Blanke et al. 2000; Luria et al. 2019). Here, we decided to implement the ESI in the most direct way in order to see, if in a clinical context, it can localize the EZ without preprocessing signal analysis.

The second limitation is related to the use of 64-channel compared to 128- or 256-channel EEG recordings that allow a higher spatial sampling. These 64-channel EEG recordings with taped electrodes facilitate long term sampling, required for seizure recordings. Furthermore, a previous study comparing 31-, 64-, 128- and 256-channel EEG recordings demonstrated that the most crucial step in increasing source localization accuracy was related to the increase from 31 to 64 electrodes (Lantz et al. 2003). Therefore, 64-channel EEG recordings were performed as a viable compromise between dense array and clinical practice. It is important to notice that all cohort studies in ictal ESI relied on the common 21-chanel EEG recording. The third one is related to the sampling bias of SEEG resulting from the partial coverage of the cortical surface (Kahane et al. 2006) that may affect the results of sub-lobar concordance. SEEG estimation of the EZ was chosen as the reference method, as opposed to surgical volume, because it was physiologically meaningful in: (i) validating another electrophysiological investigation, (ii) differentiating the zone of seizure initiation from the zone of propagation, (iii) including cases with a surgical contraindication for functional reasons (Rikir et al. 2014). Finally, we did not compare iESI and iiESI with SEEG estimated the irrative (primary or secondary) zone because we wanted to discuss about ESI in epilepsy surgery context that aims to localize the EZ.

Our study confirms the feasibility (Koessler et al. 2010; Medvedovsky et al. 2012; Pellegrino et al. 2016; Staljanssens et al. 2017; Nemtsas et al. 2017; Kuo et al. 2018; Koren et al. 2018; Sharma et al. 2018) of ictal recordings with 64-channel EEG as (i) only $22 \%$ (17/78) of patients did not have seizure (in the same range as Sharma et al. 2018) and (ii) for the others, a mean of 5.7 seizures per patient (range 1-30) was recorded. Nevertheless, this study does not confirm the superiority of ictal ESI by comparison to 
inter-ictal ESI. In addition, some statistical tests in the subgroup analysis were not significant due to a low number of patients. For example, the ictal pattern subgroup (ictal spikes or rhythmic discharges) should give interesting results with a larger cohort. Indeed, a recent SEEG study demonstrated the presence of a special fingerprint (combining pre-ictal spikes, fast activity and suppression) that occurs during preictal to ictal transition and that allows to clearly distinguish the epileptogenic zone from the brain areas of propagation (Grinenko et al. 2018).

\section{Conclusion}

In conclusion, this prospective study with a strictly uniform methodology does not show a better overall sensitivity and specificity of ictal compared to inter-ictal ESI but demonstrates the relevance of (i) a distinct inter-ictal and ictal analyses and (ii) a combined interpretation of both electrical source localizations. Inter-ictal analysis gave the highest sensitivity whereas ictal analysis gave the highest specificity. Inter-ictal ESI would be particularly interesting in MRI negative (supposed FCD) patients while ictal analysis would be considered primarily in (i) MCD patients and/ or with an MRI lesion, and, (ii) initial spike or rhythmic discharge $>13 \mathrm{~Hz}$ as ictal EEG pattern.

Acknowledgements This study was supported by the French Ministry of Health (PHRC 17-05, 2009).

Funding Estelle Rikir was supported by a grant from the Medical Council of the CHU of Liège, Belgium.

\section{Compliance with Ethical Standards}

Conflict of interest None of the authors has any conflict of interest to disclose. We confirm that we have read the Journal's position on issues involved in ethical publication and affirm that this report is consistent with those guidelines.

\section{References}

Abdallah C, Maillard LG, Rikir E et al (2017) Localizing value of electrical source imaging: frontal lobe, malformations of cortical development and negative MRI related epilepsies are the best candidates. Neuroimage Clin 16:319-329

Alarcon G, Guy CN, Binnie CD et al (1994) Intracerebral propagation of interictal activity in partial epilepsy: implications for source localisation. J Neurol Neurosurg Psychiatry 57:435-449

Alarcon G, Binnie CD, Elwes RD, Polkey CE (1995) Power spectrum and intracranial EEG patterns at seizure onset in partial epilepsy. Electroencephalogr Clin Neurophysiol 94:326-337

Alarcon G, Garcia Seoane JJ et al (1997) Origin and propagation of interictal discharges in the acute electrocorticogram. Implications for pathophysiology and surgical treatment of temporal lobe epilepsy. Brain 120:2259-2282
Bancaud J, Angelergues R, Bernouilli C et al (1970) Functional stereotaxic exploration (SEEG) of epilepsy. Electroencephalogr Clin Neurophysiol 28(1):85-86

Bartolomei F, Trébuchon A, Bonini F et al (2016) What is the concordance between the seizure onset zone and the irritative zone? A SEEG quantified study. Clin Neurophysiol 127:1157-1162

Beniczky S, Lantz G, Rosenzweig I et al (2013) Source localization of rhythmic ictal EEG activity: a study of diagnostic accuracy following STARD criteria. Epilepsia 54:1743-1752

Blanke O, Lantz G, Seeck M et al (2000) Temporal and spatial determination of EEG-seizure onset in the frequency domain. Clin Neurophysiol 111(5):763-772

Boon P, D’Havé M, Vanrumste B et al (2002) Ictal source localization in presurgical patients with refractory epilepsy. J Clin Neurophysiol 19:461-468

Brodbeck V, Spinelli L, Lascano AM et al (2011) Electroencephalographic source imaging: a prospective study of 152 operated epileptic patients. Brain 134:2887-2897

Chassoux F, Devaux B, Landré E et al (2000) Stereoencephalography in focal cortical dysplasia: a 3D approach to delineating the dysplastic cortex. Brain 123:1733-1751

Duncan JS (2003) Neuroimaging in epilepsy: quality and not just quantity is important: current resources for neuroimaging could be used more efficiently. J Neurol Neurosurg Psychiatry 73:612-613

Foldvary N, Klem G, Hammel J et al (2001) The localizing value of ictal EEG in focal epilepsy. Neurology 57:2022-2028

Fuchs M, Wagner M, Kastner J (2004) Confidence limits of dipole source reconstruction results. Clin Neurophysiol 115:1442-1451

Gavaret M, Badier JM, Marquis P et al (2004) Electric source imaging in temporal lobe epilepsy. J Clin Neurophysiol 21:267-282

Gavaret M, Badier JM, Marquis P et al (2006) Electric source imaging in frontal lobe epilepsy. J Clin Neurophysiol 23:358-370

Gavaret M, Trébuchon A, Bartolomei F et al (2009) Source localization of scalp-EEG interictal spikes in posterior cortex epilepsies investigated by HR-EEG and SEEG. Epilepsia 50:276-289

Grinenko O, Li J, Mosher JC, Wang IZ et al (2018) A fingerprint of the epileptogenic zone in human epilepsies. Brain 141(1):117-131

Guggisberg AG, Dalal SS, Zumer JM et al (2011) Localization of cortico-peripheral coherence with electroencephalography. Neuroimage 57:1348-1357

Hämäläinen MS, Ilmoniemi RJ (1994) Interpreting measured magnetic fields of the brain: estimation of current distributions. Med Biol Eng Comput 32:35-42

Jonas J, Vignal JP, Baumann C et al (2011) Effect of hyperventilation on seizure activation: potentiation by antiepileptic drug tapering. J Neurol Neurosurg Psychiatry 82:928-930

Kahane P, Landré E, Minotti L et al (2006) The Bancaud and Talairach view on the epileptogenic zone: a working hypothesis. Epileptic Disord 8(Suppl 2):S16-26

Knowlton RC, Razdan SN, Limdi N et al (2009) Effect of epilepsy magnetic source imaging on intracranial electrode placement. Ann Neurol 65:716-723

Koessler L, Benhadid A, Maillard L et al (2008) Automatic localization and labeling of EEG sensors (ALLES) in MRI volume. Neuroimage 41:914-923

Koessler L, Maillard L, Benhadid A et al (2009) Automated cortical projection of EEG sensors: anatomical correlation via the international 10-10 system. Neuroimage 46:64-72

Koessler L, Benar C, Maillard L et al (2010) Source localization of ictal epileptic activity investigated by high resolution EEG and validated by SEEG. Neuroimage 51:642-653

Koessler L, Cecchin T, Colnat-Coulbois S et al (2015) Catching the invisible: mesial temporal source contribution to simultaneous EEG and SEEG recordings. Brain Topogr 28:5-20

Koessler L, Colnat-Coulbois S, Cecchin T et al (2017) In-vivo measurements of human brain tissue conductivity using focal electrical 
current injection through intracerebral multicontact electrodes. Hum Brain Mapp 38(2):974-986

Koren J, Gritsch G, Pirker S et al (2018) Automatic ictal onset source localization in presurgical epilepsy evaluation. Clin Neurophysiol 129(6):1291-1299

Kuo CC, Tucker DM, Luu P et al (2018) EEG source imaging of epileptic activity at seizure onset. Epilepsy Res 146:160-171

Lagarde S, Bonini F, McGonigal A et al (2016) Seizure-onset patterns in focal cortical dysplasia and neurodevelopmental tumors: relationship with surgical prognosis and neuropathologic subtypes. Epilepsia 57:1426-1435

Lantz G, Michel CM, Seeck M et al (1999) Frequency domain EEG source localization of ictal epileptiform activity in patients with partial complex epilepsy of temporal lobe origin. Clin Neurophysiol 110(1):176-184

Lascano AM, Perneger T, Vulliemoz S et al (2016) Yield of MRI, high-density electric source imaging (HD-ESI), SPECT and PET in epilepsy surgery candidates. Clin Neurophysiol 127(1):150-155

Lantz G, Grave de Peralta R, Spinelli L et al (2003) Epileptic source localization with high density EEG: how many electrodes are needed? Clin Neurophysiol 114:63-69

Lüders HO, Najm I, Nair D et al (2006) The epileptogenic zone: general principles. Epileptic Disord 8 Suppl 2:S1-9

Luria G, Duran D, Visani E (2019) Bayesian multi-dipole modelling in the frequency domain. J Neurosci Methods 312:27-36

Maillard L, Koessler L, Colnat-Coulbois S et al (2009) Combined SEEG and source localisation study of temporal lobe schizencephaly and polymicrogyria. Clin Neurophysiol 120:1628-1636

Maillard LG, Tassi L, Bartolomei F et al (2017) Stereoelectroencephalography and surgical outcome in polymicrogyria-related epilepsy: a multicentric study. Ann Neurol 82(5):781-794

Medvedovsky M, Taulu S, Gaily E et al (2012) Sensitivity and specificity of seizure-onset zone estimation by ictal magnetoencephalography. Epilepsia 53:1649-1657

Michel CM, Lantz G, Spinelli L et al (2004) 128-channel EEG source imaging in epilepsy: clinical yield and localization precision. J Clin Neurophysiol 21:71-83

Mosher JC, Lewis PS, Leahy RM (1992) Multiple dipole modeling and localization from spatio-temporal MEG data. IEEE Trans Biomed Eng 39:541-557

Nemtsas P, Birot G, Pittau F et al (2017) Source localization of ictal epileptic activity based on high-density scalp EEG data. Epilepsia 58(6):1027-1036

Oostenveld R, Praamstra P (2001) The five percent electrode system for high-resolution EEG and ERP measurements. Clin Neurophysiol 112:713-719

Papayannis CE, Consalvo D, Kauffman MA et al (2012) Malformations of cortical development and epilepsy in adult patients. Seizure $21: 377-384$
Pascual-Marqui RD (2002) Standardized low-resolution brain electromagnetic tomography (sLORETA): technical details. Methods Find Exp Clin Pharmacol 24(Suppl D):S5-12

Pellegrino G, Hedrich T, Chowdhury R et al (2016) Source localization of the seizure onset zone from ictal EEG/MEG data. Hum Brain Mapp 37:2528-2546

Ramantani G, Koessler L, Colnat-Coulbois S et al (2013) Intracranial evaluation of the epileptogenic zone in regional infrasylvian polymicrogyria. Epilepsia 54:296-304

Rikir E, Koessler L, Gavaret M et al (2014) Electrical source imaging in cortical malformation-related epilepsy: a prospective EEGSEEG concordance study. Epilepsia 55:918-932

Rikir E, Koessler L, Ramantani G, Maillard LG (2017) Added value and limitations of electrical source localization. Epilepsia 58:174-175

Salado AL, Koessler L, De Mijolla G et al (2018) sEEG is a safe procedure for a comprehensive anatomic exploration of the insula: a retrospective study of 108 procedures representing 254 transopercular insular electrodes. Oper Neurosurg 14:1-8

Scherg M (1990) Fundamentals of dipole source potential analysis. In: Grandori F, Hoke M, Romani GL (eds) Auditory evoked magnetic fields and electric potentials (Advance in audiology). Karger, Basel, pp 40-69

Seeck M, Koessler L, Bast T et al (2017) The standardized EEG electrode array of the IFCN. Clin Neurophysiol 128:2070-2077

Sharma P, Scherg M, Pinborg LH et al (2018) Ictal and interictal electric source imaging in pre-surgical evaluation: a prospective study. Eur J Neurol 25(9):1154-1160

So N, Gotman J (1990) Changes in seizure activity following anticonvulsant drug withdrawal. Neurology 40:407-413

Spencer S, Huh L (2008) Outcomes of epilepsy surgery in adults and children. Lancet Neurol 7:525-537

Staljanssens W, Strobbe G, Holen RV et al (2017) Seizure onset zone localization from ictal high-density EEG in refractory focal epilepsy. Brain Topogr 30(2):257-271

Talairach J, Bancaud J (1966) Lesion, "irritative" zone and epileptogenic focus. Confin Neurol 27:91-94

Tonini C, Beghi E, Berg AT et al (2004) Predictors of epilepsy surgery outcome: a meta-analysis. Epilepsy Res 62:75-87

Trujillo-Barreto NJ, Aubert-Vazquez E, Valdes-Sosa PA (2004) Bayesian model averaging in EEG/MEG imaging. Neuroimage 21:1300-1319

Publisher's Note Springer Nature remains neutral with regard to jurisdictional claims in published maps and institutional affiliations. 